\title{
Characterizing and Improving Reaction Times for $E$. coli-based Cell-Free Protein Synthesis
}

Logan R. Burrington ${ }^{1,2}$, Katharine R. Watts ${ }^{1,2}$, and Javin P. Oza ${ }^{1,2 *}$

${ }^{1}$ Chemistry and Biochemistry Department, California Polytechnic State University, 1 Grand Avenue, San Luis Obispo, California 93407, United States

${ }^{2}$ Center for Applications in Biotechnology, California Polytechnic State University, 1 Grand Avenue, San Luis Obispo, California 93407, United States

* Correspondence: joza@calpoly.edu; 805-765-2265

\section{Supporting Information:}

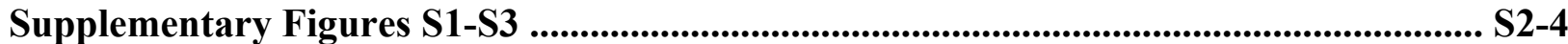

Figure S1: sfGFP titration range under ambient light and blue light $\ldots \ldots \ldots \ldots \ldots \ldots . . . . \ldots \ldots$

Figure S2: Time course data for commercial E. coli-based extract kits ........................S3

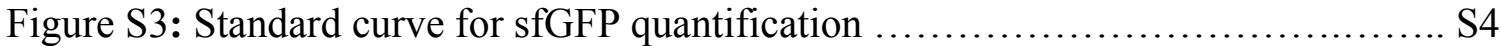

Supplementary Tables S1-S5 _.................................................................................... S5-13

Table S1: Single-additive rate analysis data with 2xYTPG extract ................. S5-6

Table S2: Multi-additive rate analysis data with 2xYTPG extract $\ldots \ldots \ldots \ldots \ldots \ldots \ldots . . . \ldots \ldots$

Table S3: Single-additive rate analysis data with CFAI OD 60010 extract ............. S8-9

Table S4: Multi-additive rate analysis data with CFAI OD 60010 extract .............. S10

Table S5: Single-additive rate analysis data with CFAI OD 6002.5 extract $\ldots \ldots \ldots \ldots \ldots$ S1 1

Table S6: Commercial E. coli-based Extract Kits Identifying Information ................. S12

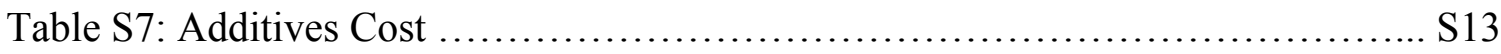

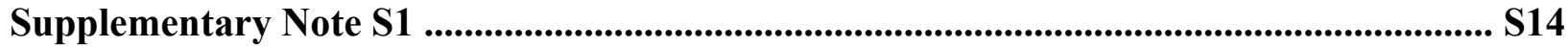

Note S1: Pre-Grubbs' Raw Data ............................................................ S14 


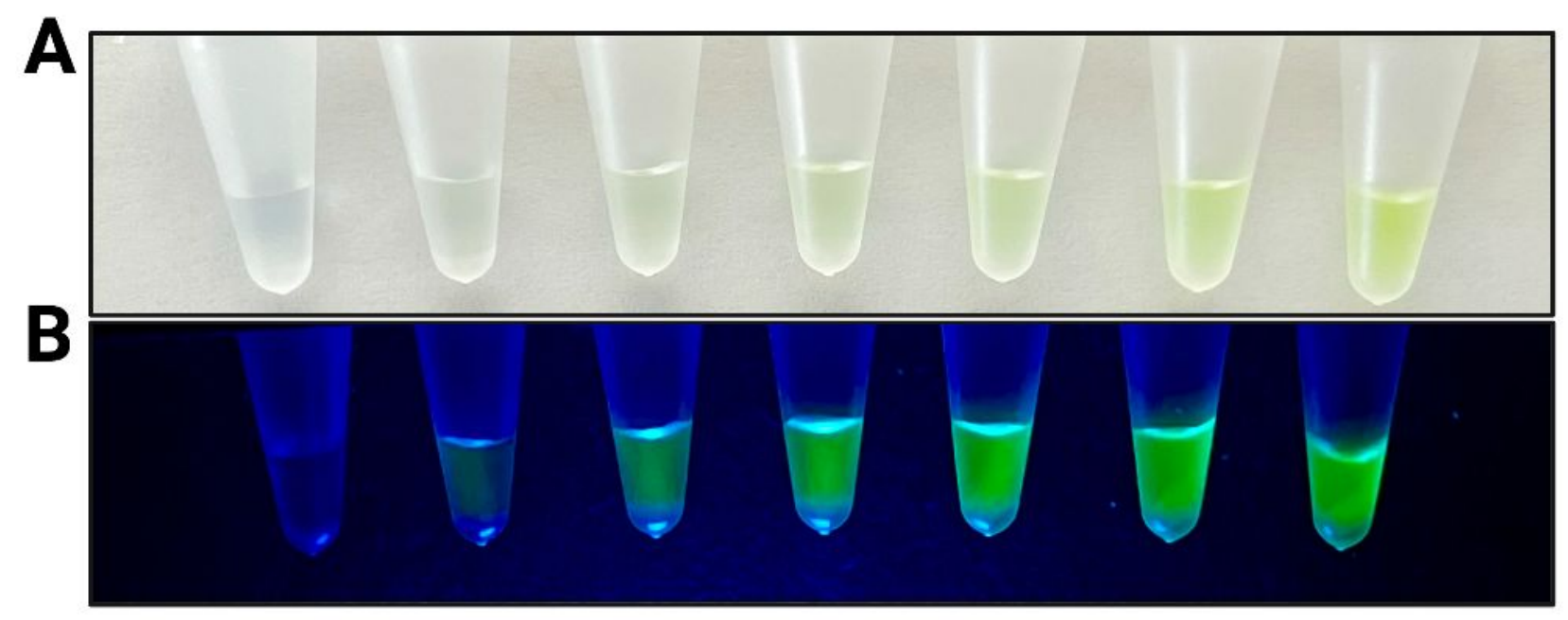

Figure S1. Titration range of sfGFP under A) ambient light and B) blue light with an orange light-filtering screen. Respective titration concentrations from left to right are: $0,50,100,150,200,250$, and $300 \mu \mathrm{g} / \mathrm{mL} \mathrm{sfGFP}$. Each tube consists of a $30 \mu \mathrm{L}$ aliquot. 
A

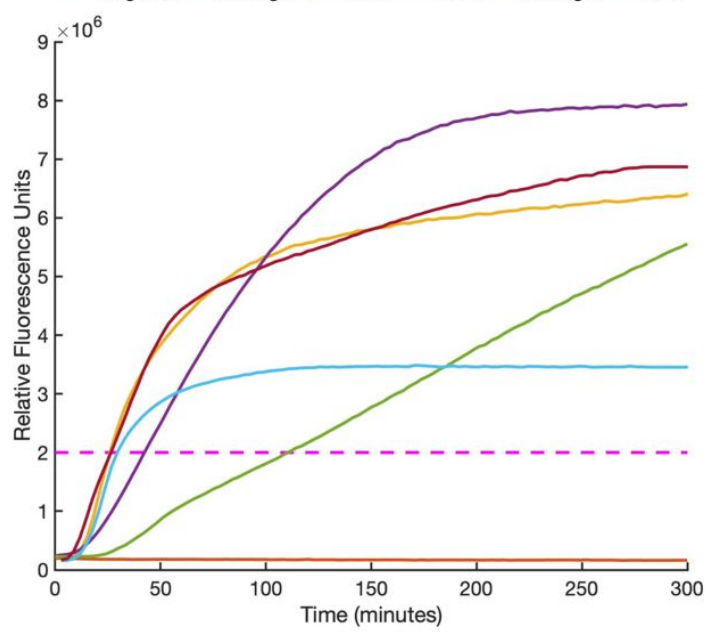

B

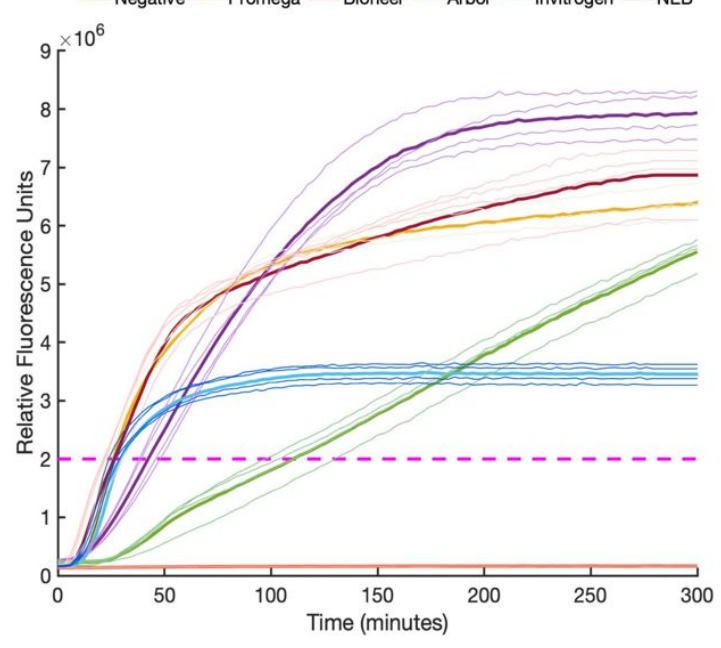

Figure S2. Time course data for commercial E. coli-based extract kits from Promega (yellow), Bioneer (purple), Arbor (green), Invitrogen (blue), and NEB (dark red). The $200 \mu \mathrm{g} / \mathrm{mL}\left(2 \times 10^{6} \mathrm{RFU}\right)$ limit of detection is depicted (dash). The negative condition (orange) has no pJL1-sfGFP DNA template present. Data is plotted as an average of a minimum of $\mathrm{N}=3$, maximum $\mathrm{N}=4$ experimental replicates $\mathbf{A}$ ) without and $\mathbf{B}$ ) with replicates included. 


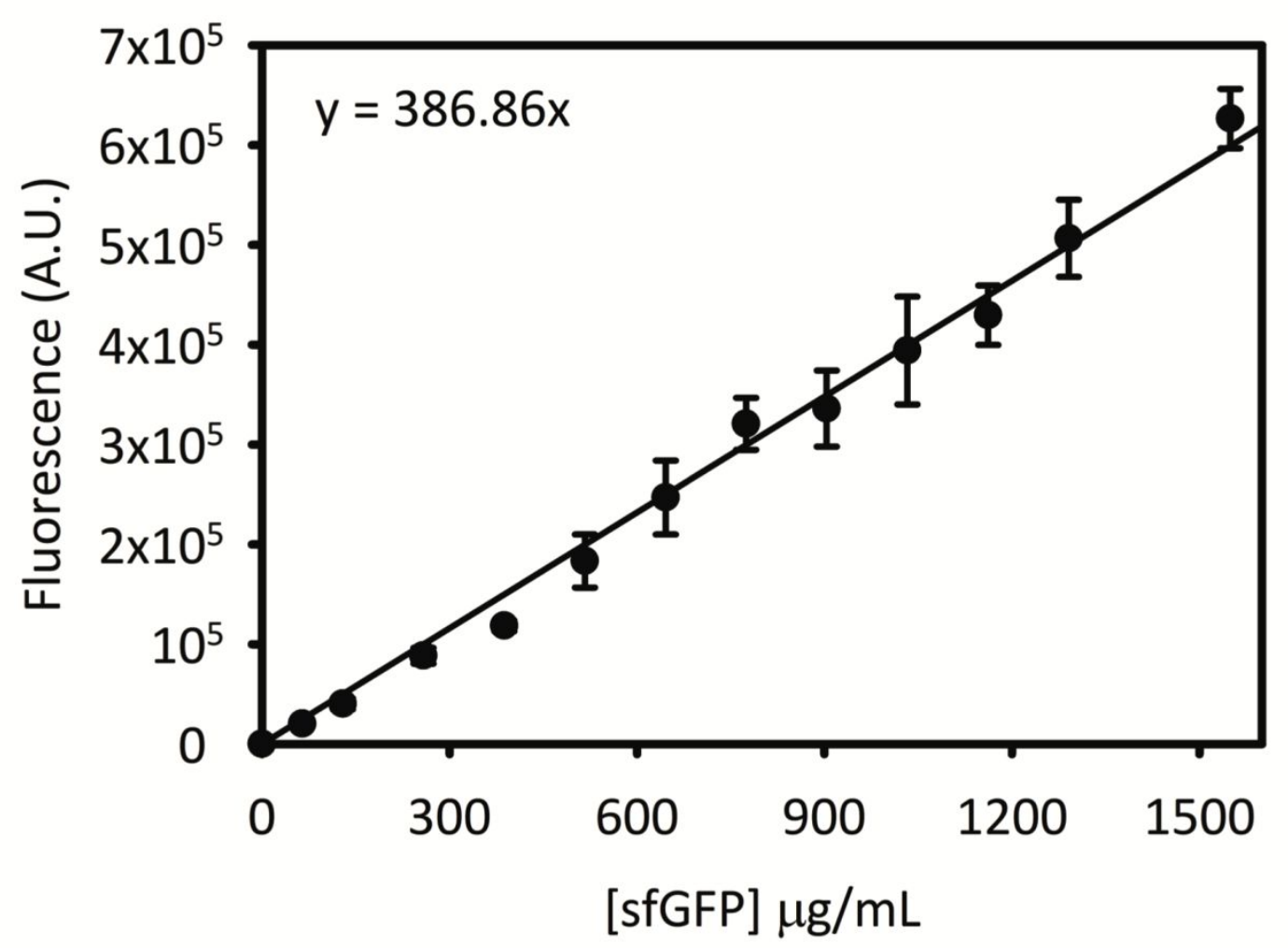

Figure S3. Standard curve for sfGFP quantification from CFPS reactions on a BioTek Cytation5. Our standard curve was generated by purifying sfGFP from an $E$. coli expression and determining the concentration using a spectrophotometer, Beer's law, and a calculated extinction coefficient based on the protein sequence. The pure sfGFP was then diluted 1:25 in the range of $0-1500 \mu \mathrm{g} / \mathrm{mL}$ in triplicate onto a 96 -well black bottom plate and the absorbance values were used to generate this standard curve. Points represent the mean of three measurements and error bars represent the standard deviation of the three measurements. 
Table S1. Single-Additive Rate Analysis Data With 2xYTPG Extract

\begin{tabular}{|c|c|c|c|c|}
\hline $\begin{array}{c}\text { Additive } \\
\text { (Conditions for positive } \\
\text { control) }\end{array}$ & Concentration & $\begin{array}{l}\text { Time to } 200 \mu \mathrm{g} / \mathrm{mL} \\
\quad \text { [sfGFP] }(\mathrm{min})\end{array}$ & $\begin{array}{l}\text { Time to } 100 \mu \mathrm{g} / \mathrm{mL} \\
\text { [sfGFP] }(\mathrm{min})\end{array}$ & Yield $(\mu \mathrm{g} / \mathrm{mL})$ \\
\hline \multirow[t]{4}{*}{$\begin{array}{c}\text { Extract } \\
(33 \% \mathrm{v} / \mathrm{v})\end{array}$} & $33 \% \mathrm{v} / \mathrm{v}$ & $44.72 \pm 2.74$ & $29.43 \pm 2.01$ & $966 \pm 56$ \\
\hline & $40 \% \mathrm{v} / \mathrm{v}$ & $35.97 \pm 0.99$ & $23.49 \pm 0.68$ & $1084 \pm 32$ \\
\hline & $47 \% \mathrm{v} / \mathrm{v}$ & $33.49 \pm 2.8$ & $20.9 \pm 1.67$ & $1086 \pm 54$ \\
\hline & $53 \% \mathrm{v} / \mathrm{v}$ & $33.34 \pm 2.6$ & $20.48 \pm 0.85$ & $1152 \pm 85$ \\
\hline \multirow[t]{4}{*}{$\begin{array}{c}\text { ATP } \\
(1.2 \mathrm{mM})\end{array}$} & $1.8 \mathrm{mM}$ & $32.32 \pm 2.01$ & $21.16 \pm 0.41$ & $988 \pm 73$ \\
\hline & $2.1 \mathrm{mM}$ & $27.58 \pm 2.02$ & $19.39 \pm 0.92$ & $1115 \pm 93$ \\
\hline & $2.4 \mathrm{mM}$ & $27.08 \pm 2.42$ & $19.86 \pm 1.90$ & $1192 \pm 104$ \\
\hline & $2.7 \mathrm{mM}$ & $28.47 \pm 2.71$ & $19.54 \pm 0.93$ & $1180 \pm 88$ \\
\hline \multirow[t]{3}{*}{$\begin{array}{c}\text { GTP } \\
(0.85 \mathrm{mM})\end{array}$} & $1.24 \mathrm{mM}$ & $42.31 \pm 5.85$ & $24.00 \pm 3.88$ & $954 \pm 95$ \\
\hline & $1.62 \mathrm{mM}$ & $42.44 \pm 2.64$ & $25.05 \pm 1.14$ & $905 \pm 30$ \\
\hline & $2.01 \mathrm{mM}$ & $48.45 \pm 4.80$ & $27.45 \pm 2.69$ & $959 \pm 47$ \\
\hline \multirow[t]{3}{*}{$\begin{array}{c}\text { PEP } \\
(33 \mathrm{mM})\end{array}$} & $41.25 \mathrm{mM}$ & $37.32 \pm 2.28$ & $26.63 \pm 1.06$ & $1143 \pm 61$ \\
\hline & $49.5 \mathrm{mM}$ & $36.75 \pm 1.62$ & $26.81 \pm 1.62$ & $1074 \pm 142$ \\
\hline & $57.75 \mathrm{mM}$ & $39.90 \pm 6.23$ & $29.25 \pm 4.23$ & $1187 \pm 16$ \\
\hline \multirow[t]{4}{*}{$\begin{array}{l}\text { PEG8k } \\
(0 \% \mathrm{w} / \mathrm{v})\end{array}$} & $0.1 \% \mathrm{w} / \mathrm{v}$ & $34.91 \pm 2.19$ & $24.89 \pm 1.06$ & $1069 \pm 31$ \\
\hline & $0.3 \% \mathrm{w} / \mathrm{v}$ & $33.79 \pm 1.27$ & $24.04 \pm 0.95$ & $1121 \pm 40$ \\
\hline & $0.5 \% \mathrm{w} / \mathrm{v}$ & $30.19 \pm 1.15$ & $22.2 \pm 0.58$ & $1125 \pm 67$ \\
\hline & $0.7 \% \mathrm{w} / \mathrm{v}$ & $30.09 \pm 1.39$ & $22.34 \pm 1.06$ & $1042 \pm 48$ \\
\hline \multirow[t]{5}{*}{$\begin{array}{c}\mathrm{TX}-100 \\
(0 \mathrm{CMC})^{* *}\end{array}$} & $15 \mathrm{CMC}$ & $35.22 \pm 0.69$ & $24.89 \pm 0.78$ & $384 \pm 50$ \\
\hline & $30 \mathrm{CMC}$ & $33.53 \pm 1.71$ & $23.71 \pm 1.02$ & $373 \pm 7$ \\
\hline & $50 \mathrm{CMC}$ & $26.87 \pm 2.18$ & $18.23 \pm 1.59$ & $419 \pm 36$ \\
\hline & $75 \mathrm{CMC}$ & $27.70 \pm 2.12$ & $18.77 \pm 2.00$ & $401 \pm 39$ \\
\hline & $100 \mathrm{CMC}$ & $27.30 \pm 0.66$ & $18.34 \pm 0.62$ & $419 \pm 20$ \\
\hline $\begin{array}{l}\text { Maltose } \\
(0 \mathrm{mM})\end{array}$ & $15 \mathrm{mM}$ & $39.64 \pm 5.13$ & $28.15 \pm 3.23$ & $\mathrm{~N} / \mathrm{A}$ \\
\hline
\end{tabular}


* All values are the post-Grub's test mean of a minimum of N=3 and a maximum of $\mathrm{N}=4$ experimental replicates \pm the standard deviation.

* All listed concentrations represent concentrations in the final $30 \mu \mathrm{L}$ CFPS reaction.

* All additives titrations (subsequent to extract titrations) are performed with $40 \% \mathrm{v} / \mathrm{v}$ cell extract

* Positive controls are highlighted in green

* Best conditions are highlighted in yellow

** $1 \mathrm{CMC}=0.23 \mathrm{mM}$ TX-100 
Table S2. Multi-Additive Rate Analysis Data With 2xYTPG Extract

\begin{tabular}{|c|c|c|c|c|c|}
\hline Additives & Concentration & $\begin{array}{c}\text { Time to } 200 \\
\mu \mathrm{g} / \mathrm{mL}[\mathrm{sfGFP}] \\
\underset{\text { (min) }}{ }\end{array}$ & $\begin{array}{l}\text { Time to } 100 \\
\mu \mathrm{g} / \mathrm{mL}[\mathrm{sfGFP}] \\
\underset{\text { (min) }}{\text { min }}\end{array}$ & Yield $(\mu \mathrm{g} / \mathrm{mL})$ & Shaking? \\
\hline \multirow[t]{2}{*}{ Extract } & $33 \% \mathrm{v} / \mathrm{v}$ & $44.72 \pm 2.74$ & $29.43 \pm 2.01$ & $966 \pm 56$ & $\mathrm{~N}$ \\
\hline & $40 \% \mathrm{v} / \mathrm{v}$ & $35.97 \pm 0.99$ & $23.49 \pm 0.68$ & $1084 \pm 32$ & $\mathrm{~N}$ \\
\hline $\begin{array}{l}\text { Extract } \\
\text { ATP } \\
\text { PEG8k }\end{array}$ & $\begin{array}{c}40 \% \mathrm{v} / \mathrm{v} \\
2.4 \mathrm{mM} \\
0.5 \% \mathrm{w} / \mathrm{v}\end{array}$ & $23.59 \pm 2.54$ & $15.75 \pm 1.91$ & $932 \pm 50$ & $\mathrm{~N}$ \\
\hline $\begin{array}{l}\text { Extract } \\
\text { ATP } \\
\text { PEG8k }\end{array}$ & $\begin{array}{c}40 \% \mathrm{v} / \mathrm{v} \\
2.4 \mathrm{mM} \\
0.5 \% \mathrm{w} / \mathrm{v}\end{array}$ & $16.99 \pm 0.48$ & $12.20 \pm 0.37$ & $1015 \pm 51$ & $\mathrm{Y}$ \\
\hline $\begin{array}{l}\text { Extract } \\
\text { ATP } \\
\text { PEG8k } \\
\text { TX-100 }\end{array}$ & $\begin{array}{c}40 \% \mathrm{v} / \mathrm{v} \\
2.4 \mathrm{mM} \\
0.5 \% \mathrm{w} / \mathrm{v} \\
50 \mathrm{CMC}^{* *}\end{array}$ & $19.75 \pm 1.37$ & $13.26 \pm 0.78$ & $325 \pm 51$ & $\mathrm{~N}$ \\
\hline $\begin{array}{l}\text { Extract } \\
\text { ATP } \\
\text { PEG8k } \\
\text { TX-100 }\end{array}$ & $\begin{array}{c}40 \% \mathrm{v} / \mathrm{v} \\
2.4 \mathrm{mM} \\
0.5 \% \mathrm{w} / \mathrm{v} \\
50 \mathrm{CMC}^{* *}\end{array}$ & $14.94 \pm 0.84$ & $9.91 \pm 0.58$ & $419 \pm 27$ & $\mathrm{Y}$ \\
\hline $\begin{array}{c}\text { Extract } \\
\text { ATP } \\
\text { PEG8k } \\
\mathrm{Mg}(\mathrm{GLU})_{2}\end{array}$ & $\begin{array}{c}40 \% \mathrm{v} / \mathrm{v} \\
2.4 \mathrm{mM} \\
0.5 \% \mathrm{w} / \mathrm{v} \\
14 \mathrm{mM}\end{array}$ & $26.26 \pm 2.14$ & $18.76 \pm 2.72$ & $938 \pm 49$ & $\mathrm{~N}$ \\
\hline $\begin{array}{c}\text { Extract } \\
\text { ATP } \\
\text { PEG8k } \\
\mathrm{Mg}(\mathrm{GLU})_{2}\end{array}$ & $\begin{array}{c}40 \% \mathrm{v} / \mathrm{v} \\
2.4 \mathrm{mM} \\
0.5 \% \mathrm{w} / \mathrm{v} \\
16 \mathrm{mM}\end{array}$ & $33.68 \pm 3.92$ & $19.50 \pm 2.55$ & $899 \pm 55$ & $\mathrm{~N}$ \\
\hline $\begin{array}{l}\text { Extract } \\
\text { ATP } \\
\text { PEG8k } \\
\text { T7 RNAP }\end{array}$ & $\begin{array}{c}40 \% \mathrm{v} / \mathrm{v} \\
2.4 \mathrm{mM} \\
0.5 \% \mathrm{w} / \mathrm{v} \\
1 \% \mathrm{v} / \mathrm{v}\end{array}$ & $29.41 \pm 1.96$ & $20.20 \pm 2.54$ & $848 \pm 24$ & $\mathrm{~N}$ \\
\hline
\end{tabular}

* All values are the post-Grub's test mean of a minimum of $\mathrm{N}=3$ and a maximum of $\mathrm{N}=4$ experimental replicates \pm the standard deviation.

* All listed concentrations represent concentrations in the final $30 \mu \mathrm{L}$ CFPS reaction.

* Positive controls are highlighted in green

$* * 50 \mathrm{CMC}=11.50 \mathrm{mM}$ TX-100 
Table S3. Single-Additive Rate Analysis Data With CFAI OD 600 Extract

\begin{tabular}{|c|c|c|c|c|}
\hline $\begin{array}{c}\text { Additive } \\
\text { (Control Concentration) }\end{array}$ & Concentration & $\begin{array}{l}\text { Time to } 200 \mu \mathrm{g} / \mathrm{mL} \\
\quad[\mathrm{sfGFP}](\mathrm{min})\end{array}$ & $\begin{array}{l}\text { Time to } 100 \mu \mathrm{g} / \mathrm{mL} \\
\text { [sfGFP] }(\mathrm{min})\end{array}$ & Yield $(\mu \mathrm{g} / \mathrm{mL})$ \\
\hline \multirow[t]{4}{*}{$\begin{array}{c}\text { Extract } \\
(33 \% \mathrm{v} / \mathrm{v})\end{array}$} & $33 \% \mathrm{v} / \mathrm{v}$ & $84.18 \pm 3.90$ & $52.33 \pm 0.74$ & $915 \pm 23$ \\
\hline & $40 \% \mathrm{v} / \mathrm{v}$ & $58.17 \pm 4.32$ & $37.09 \pm 2.37$ & $1055 \pm 41$ \\
\hline & $47 \% \mathrm{v} / \mathrm{v}$ & $60.37 \pm 4.38$ & $35.14 \pm 2.97$ & $1075 \pm 55$ \\
\hline & $53 \% \mathrm{v} / \mathrm{v}$ & $51.72 \pm 1.42$ & $25.65 \pm 4.83$ & $1063 \pm 63$ \\
\hline \multirow[t]{7}{*}{$\begin{array}{c}\text { Plasmid } \\
(16 \mathrm{ng} / \mu \mathrm{L})\end{array}$} & $10 \mathrm{ng} / \mu \mathrm{L}$ & $62.80 \pm 5.53$ & $40.28 \pm 4.31$ & $940 \pm 92$ \\
\hline & $12 \mathrm{ng} / \mu \mathrm{L}$ & $69.81 \pm 8.30$ & $43.25 \pm 4.37$ & $931 \pm 62$ \\
\hline & $14 \mathrm{ng} / \mu \mathrm{L}$ & $77.21 \pm 10.74$ & $46.62 \pm 4.38$ & $946 \pm 82$ \\
\hline & $20 \mathrm{ng} / \mu \mathrm{L}$ & $59.11 \pm 7.31$ & $38.40 \pm 1.87$ & $1155 \pm 111$ \\
\hline & $24 \mathrm{ng} / \mu \mathrm{L}$ & $71.31 \pm 14.91$ & $41.80 \pm 2.81$ & $1203 \pm 92$ \\
\hline & $28 \mathrm{ng} / \mu \mathrm{L}$ & $63.59 \pm 5.85$ & $39.64 \pm 0.51$ & $1120 \pm 129$ \\
\hline & $32 \mathrm{ng} / \mu \mathrm{L}$ & $80.13 \pm 6.89$ & $52.6 \pm 2.43$ & $1015 \pm 46$ \\
\hline \multirow[t]{4}{*}{$\begin{array}{c}\text { ATP } \\
(1.2 \mathrm{mM})\end{array}$} & $1.5 \mathrm{mM}$ & $58.17 \pm 3.22$ & $38.68 \pm 1.45$ & $1148 \pm 16$ \\
\hline & $1.8 \mathrm{mM}$ & $63.00 \pm 6.25$ & $40.14 \pm 2.07$ & $1166 \pm 50$ \\
\hline & $2.1 \mathrm{mM}$ & $55.93 \pm 4.38$ & $36.13 \pm 1.24$ & $1184 \pm 33$ \\
\hline & $2.4 \mathrm{mM}$ & $45.97 \pm 5.08$ & $32.44 \pm 3.27$ & $1234 \pm 16$ \\
\hline \multirow[t]{4}{*}{$\begin{array}{c}\mathrm{GTP} \\
(0.85 \mathrm{mM})\end{array}$} & $1.24 \mathrm{mM}$ & $65.64 \pm 5.36$ & $38.55 \pm 3.10$ & $940 \pm 49$ \\
\hline & $1.62 \mathrm{mM}$ & $66.48 \pm 10.58$ & $39.25 \pm 5.03$ & $980 \pm 137$ \\
\hline & $2.01 \mathrm{mM}$ & $59.10 \pm 5.32$ & $36.83 \pm 1.69$ & $926 \pm 60$ \\
\hline & $2.40 \mathrm{mM}$ & $57.62 \pm 5.14$ & $34.97 \pm 2.71$ & $972 \pm 67$ \\
\hline \multirow[t]{4}{*}{$\begin{array}{c}\text { PEP } \\
(33 \mathrm{mM})\end{array}$} & $41.25 \mathrm{mM}$ & $57.75 \pm 1.06$ & $35.79 \pm 1.81$ & $859 \pm 236$ \\
\hline & $49.50 \mathrm{mM}$ & $74.45 \pm 4.72$ & $42.21 \pm 4.49$ & $897 \pm 193$ \\
\hline & $57.75 \mathrm{mM}$ & $66.55 \pm 4.65$ & $40.55 \pm 0.76$ & $945 \pm 159$ \\
\hline & $66.00 \mathrm{mM}$ & $89.90 \pm 6.64$ & $50.04 \pm 6.51$ & $900 \pm 48$ \\
\hline \multirow[t]{3}{*}{$\begin{array}{c}\text { PEG8k } \\
(0 \% \mathrm{w} / \mathrm{v})\end{array}$} & $0.1 \% \mathrm{w} / \mathrm{v}$ & $56.01 \pm 4.57$ & $25.94 \pm 0.71$ & $1050 \pm 58$ \\
\hline & $0.3 \% \mathrm{w} / \mathrm{v}$ & $56.93 \pm 4.74$ & $25.83 \pm 3.04$ & $1024 \pm 101$ \\
\hline & $0.5 \% \mathrm{w} / \mathrm{v}$ & $42.32 \pm 0.96$ & $21.54 \pm 1.00$ & $1022 \pm 70$ \\
\hline
\end{tabular}




\begin{tabular}{|c|c|c|c|c|}
\hline & $0.7 \% \mathrm{w} / \mathrm{v}$ & $56.92 \pm 7.17$ & $28.42 \pm 2.69$ & $1017 \pm 46$ \\
\hline & $0.9 \% \mathrm{w} / \mathrm{v}$ & $52.58 \pm 4.76$ & $25.23 \pm 0.51$ & $1034 \pm 63$ \\
\hline \multirow[t]{6}{*}{$\begin{array}{c}\mathrm{TX}-100 \\
(0 \mathrm{CMC})^{* *}\end{array}$} & $0.5 \mathrm{CMC}$ & $69.37 \pm 3.69$ & $39.36 \pm 0.43$ & $1071 \pm 43$ \\
\hline & $5 \mathrm{CMC}$ & $64.34 \pm 4.59$ & $35.02 \pm 2.45$ & $424 \pm 23$ \\
\hline & $15 \mathrm{CMC}$ & $60.56 \pm 4.24$ & $33.25 \pm 1.06$ & $386 \pm 18$ \\
\hline & $30 \mathrm{CMC}$ & $55.86 \pm 3.02$ & $33.70 \pm 2.26$ & $327 \pm 17$ \\
\hline & $50 \mathrm{CMC}$ & $61.58 \pm 6.76$ & $35.13 \pm 1.86$ & $300 \pm 9$ \\
\hline & $100 \mathrm{CMC}$ & $60.65 \pm 10.4$ & $34.82 \pm 4.13$ & $302 \pm 33$ \\
\hline \multirow[t]{3}{*}{$\begin{array}{l}\text { Maltose } \\
(0 \mathrm{mM})\end{array}$} & $15 \mathrm{mM}$ & $72.80 \pm 6.83$ & $42.15 \pm 0.83$ & $1252 \pm 62$ \\
\hline & $30 \mathrm{mM}$ & $256 \pm ?$ & $92.15 \pm 28.49$ & $570 \pm 101$ \\
\hline & $45 \mathrm{mM}$ & - & $64.23 \pm 10.5$ & $494 \pm 48$ \\
\hline \multirow[t]{4}{*}{$\begin{array}{l}\text { T7 RNAP } \\
(0 \% \mathrm{v} / \mathrm{v})\end{array}$} & $0.33 \% \mathrm{v} / \mathrm{v}$ & $64.87 \pm 3.70$ & $38.39 \pm 3.08$ & $1462 \pm 65$ \\
\hline & $0.66 \% \mathrm{v} / \mathrm{v}$ & $64.58 \pm 2.30$ & $36.12 \pm 0.98$ & $1295 \pm 88$ \\
\hline & $1 \% \mathrm{v} / \mathrm{v}$ & $60.10 \pm 8.71$ & $33.78 \pm 3.50$ & $1191 \pm 115$ \\
\hline & $1.33 \% \mathrm{v} / \mathrm{v}$ & $59.82 \pm 7.25$ & $34.36 \pm 2.45$ & $1121 \pm 50$ \\
\hline \multirow[t]{2}{*}{$\begin{array}{l}\text { Trehalose/Betaine } \\
\qquad(0 / 0 \mathrm{mM})\end{array}$} & $20 / 330 \mathrm{mM}$ & $63.68 \pm 2.94$ & $43.68 \pm 2.14$ & $1443 \pm 151$ \\
\hline & $30 / 250 \mathrm{mM}$ & $65.45 \pm 4.74$ & $44.41 \pm 3.13$ & $1051 \pm 123$ \\
\hline
\end{tabular}

* All values are the post-Grub's test mean of a minimum of $\mathrm{N}=3$ and a maximum of $\mathrm{N}=4$ experimental replicates \pm the standard deviation.

* All listed concentrations represent concentrations in the final $30 \mu \mathrm{L}$ CFPS reaction.

* “." represents conditions that did not reach the respective sfGFP concentration during the 5-hour time course reading.

* “?” represents standard deviations cannot be determined (1 or more replicates did not reach the respective concentration in time).

* All additives titrations (subsequent to extract titrations) are performed with $40 \% \mathrm{v} / \mathrm{v}$ cell extract

* Positive controls are highlighted in green

* Best conditions are highlighted in yellow

$* * 1 \mathrm{CMC}=0.23 \mathrm{mM}$ TX-100 
Table S4. Multi-Additive Rate Analysis Data With CFAI OD 60010 Extract

\begin{tabular}{|c|c|c|c|c|c|}
\hline Additives & Concentration & $\begin{array}{c}\text { Time to } 200 \\
\mu \mathrm{g} / \mathrm{mL}[\mathrm{sfGFP}] \\
\underset{\text { (min) }}{ }\end{array}$ & $\begin{array}{l}\text { Time to } 100 \\
\mu \mathrm{g} / \mathrm{mL}[\mathrm{sfGFP}] \\
\quad \text { (min) }\end{array}$ & Yield $(\mu \mathrm{g} / \mathrm{mL})$ & Shaking? \\
\hline \multirow[t]{2}{*}{ Extract } & $33 \% \mathrm{v} / \mathrm{v}$ & $84.18 \pm 3.90$ & $52.33 \pm 0.74$ & $915 \pm 23$ & $\mathrm{~N}$ \\
\hline & $40 \% \mathrm{v} / \mathrm{v}$ & $58.17 \pm 4.32$ & $37.09 \pm 2.37$ & $1055 \pm 41$ & $\mathrm{~N}$ \\
\hline $\begin{array}{l}\text { Extract } \\
\text { ATP } \\
\text { PEG8k }\end{array}$ & $\begin{array}{c}40 \% \mathrm{v} / \mathrm{v} \\
2.4 \mathrm{mM} \\
0.5 \% \mathrm{w} / \mathrm{v}\end{array}$ & $33.79 \pm 4.22$ & $23.89 \pm 1.93$ & $1201 \pm 30$ & $\mathrm{~N}$ \\
\hline $\begin{array}{l}\text { Extract } \\
\text { ATP } \\
\text { PEG8k }\end{array}$ & $\begin{array}{c}40 \% \mathrm{v} / \mathrm{v} \\
2.4 \mathrm{mM} \\
0.5 \% \mathrm{w} / \mathrm{v}\end{array}$ & $31.44 \pm 0.61$ & $14.67 \pm 0.53$ & $1240 \pm 64$ & $\mathrm{Y}$ \\
\hline $\begin{array}{c}\text { Extract } \\
\text { ATP } \\
\text { PEG8k } \\
\mathrm{Mg}(\mathrm{GLU})_{2}\end{array}$ & $\begin{array}{c}40 \% \mathrm{v} / \mathrm{v} \\
2.4 \mathrm{mM} \\
0.5 \% \mathrm{w} / \mathrm{v} \\
14 \mathrm{mM}\end{array}$ & $40.93 \pm 4.69$ & $19.54 \pm 2.73$ & $1051 \pm 55$ & $\mathrm{~N}$ \\
\hline $\begin{array}{c}\text { Extract } \\
\text { ATP } \\
\text { PEG8k } \\
\mathrm{Mg}(\mathrm{GLU})_{2}\end{array}$ & $\begin{array}{c}40 \% \mathrm{v} / \mathrm{v} \\
2.4 \mathrm{mM} \\
0.5 \% \mathrm{w} / \mathrm{v} \\
16 \mathrm{mM}\end{array}$ & $40.50 \pm 3.11$ & $21.33 \pm 4.27$ & $1022 \pm 111$ & $\mathrm{~N}$ \\
\hline $\begin{array}{l}\text { Extract } \\
\text { ATP } \\
\text { PEG8k } \\
\text { T7 RNAP }\end{array}$ & $\begin{array}{c}40 \% \mathrm{v} / \mathrm{v} \\
2.4 \mathrm{mM} \\
0.5 \% \mathrm{w} / \mathrm{v} \\
1 \% \mathrm{v} / \mathrm{v}\end{array}$ & $59.13 \pm 3.14$ & $25.07 \pm 2.19$ & $716 \pm 53$ & $\mathrm{~N}$ \\
\hline
\end{tabular}

* All values are the post-Grub's test mean of a minimum of $\mathrm{N}=3$ and a maximum of $\mathrm{N}=4$ experimental replicates \pm the standard deviation.

* All listed concentrations represent concentrations in the final $30 \mu \mathrm{L}$ CFPS reaction.

* Positive controls are highlighted in green 
Table S5. Single-Additive Rate Analysis Data With CFAI OD 600 2.5 Extract

\begin{tabular}{|c|c|c|c|c|}
\hline $\begin{array}{c}\text { Additive } \\
\text { (Control Concentration) }\end{array}$ & Concentration & $\begin{array}{l}\text { Time to } 200 \mu \mathrm{g} / \mathrm{mL} \\
\quad \text { [sfGFP] }(\mathrm{min})\end{array}$ & $\begin{array}{l}\text { Time to } 100 \mu \mathrm{g} / \mathrm{mL} \\
\text { [sfGFP] }(\mathrm{min})\end{array}$ & Yield $(\mu \mathrm{g} / \mathrm{mL})$ \\
\hline \multirow[t]{4}{*}{$\begin{array}{l}\text { Extract } \\
(33 \% \mathrm{v} / \mathrm{v})\end{array}$} & $33 \% \mathrm{v} / \mathrm{v}$ & $60.37 \pm 2.37$ & $39.38 \pm 1.78$ & $906 \pm 58$ \\
\hline & $40 \% \mathrm{v} / \mathrm{v}$ & $50.72 \pm 3.33$ & $32.16 \pm 2.31$ & $909 \pm 39$ \\
\hline & $47 \% \mathrm{v} / \mathrm{v}$ & $50.08 \pm 5.06$ & $29.39 \pm 2.82$ & $942 \pm 18$ \\
\hline & $53 \% \mathrm{v} / \mathrm{v}$ & $46.08 \pm 1.2$ & $26.56 \pm 0.80$ & $800 \pm 25$ \\
\hline \multirow[t]{3}{*}{$\begin{array}{c}\text { ATP } \\
(1.2 \mathrm{mM})\end{array}$} & $1.8 \mathrm{mM}$ & $53.77 \pm 4.68$ & $35.51 \pm 2.30$ & $904 \pm 58$ \\
\hline & $2.1 \mathrm{mM}$ & $56.57 \pm 6.20$ & $37.30 \pm 2.84$ & $782 \pm 70$ \\
\hline & $2.4 \mathrm{mM}$ & $60.81 \pm 4.96$ & $38.10 \pm 3.06$ & $817 \pm 73$ \\
\hline \multirow[t]{4}{*}{$\begin{array}{c}\text { GTP } \\
(0.85 \mathrm{mM})\end{array}$} & $1.24 \mathrm{mM}$ & $50.87 \pm 3.45$ & $33.82 \pm 2.65$ & $860 \pm 51$ \\
\hline & $1.62 \mathrm{mM}$ & $53.93 \pm 3.14$ & $35.65 \pm 1.21$ & $919 \pm 55$ \\
\hline & $2.01 \mathrm{mM}$ & $55.28 \pm 1.21$ & $36.39 \pm 1.00$ & $976 \pm 40$ \\
\hline & $2.4 \mathrm{mM}$ & $53.23 \pm 3.40$ & $35.37 \pm 2.11$ & $1063 \pm 33$ \\
\hline \multirow[t]{4}{*}{$\begin{array}{c}\text { PEP } \\
(33 \mathrm{mM})\end{array}$} & $41.25 \mathrm{mM}$ & $49.63 \pm 5.01$ & $34.06 \pm 2.44$ & $971 \pm 91$ \\
\hline & $49.5 \mathrm{mM}$ & $59.25 \pm 2.33$ & $39.78 \pm 1.77$ & $830 \pm 66$ \\
\hline & $57.75 \mathrm{mM}$ & $71.88 \pm 5.72$ & $47.70 \pm 3.05$ & $814 \pm 68$ \\
\hline & $66 \mathrm{mM}$ & $102.95 \pm 7.81$ & $67.20 \pm 5.61$ & $734 \pm 70$ \\
\hline \multirow[t]{3}{*}{$\begin{array}{l}\text { PEG8k } \\
(0 \% \mathrm{w} / \mathrm{v})\end{array}$} & $0.3 \% \mathrm{w} / \mathrm{v}$ & $48.76 \pm 3.87$ & $30.86 \pm 1.57$ & $859 \pm 52$ \\
\hline & $0.5 \% \mathrm{w} / \mathrm{v}$ & $47.76 \pm 2.63$ & $29.89 \pm 1.20$ & $838 \pm 56$ \\
\hline & $0.7 \% \mathrm{w} / \mathrm{v}$ & $46.92 \pm 1.85$ & $29.93 \pm 1.89$ & $893 \pm 34$ \\
\hline \multirow[t]{4}{*}{$\begin{array}{c}\mathrm{TX}-100 \\
(0 \mathrm{CMC})^{* *}\end{array}$} & $30 \mathrm{CMC}$ & $95.58 \pm 28.04$ & $36.58 \pm 5.70$ & $375 \pm 32$ \\
\hline & $50 \mathrm{CMC}$ & $65.60 \pm 5.68$ & $32.69 \pm 3.03$ & $387 \pm 57$ \\
\hline & $75 \mathrm{CMC}$ & $63.19 \pm 20.11$ & $35.41 \pm 6.41$ & $382 \pm 25$ \\
\hline & $100 \mathrm{CMC}$ & $191.9 \pm 77.20$ & $38.82 \pm 4.66$ & $370 \pm 13$ \\
\hline
\end{tabular}

* All values are the post-Grub's test mean of a minimum of $\mathrm{N}=3$ and a maximum of $\mathrm{N}=4$ experimental replicates \pm the standard deviation.

* All listed concentrations represent concentrations in the final $30 \mu \mathrm{L} \mathrm{CFPS} \mathrm{reaction.}$

* All additives titrations (subsequent to extract titrations) are performed with $40 \% \mathrm{v} / \mathrm{v}$ cell extract

* Positive controls are highlighted in green

$* * 1 \mathrm{CMC}=0.23 \mathrm{mM}$ TX-100 
Table S6. Commercial E. coli-based Extract Kits Identifying Information

\begin{tabular}{|c|c|c|c|c|c|}
\hline Company & Arbor & Bioneer & Invitrogen & NEB & Promega \\
\hline Kit & MyTxTl & $\begin{array}{c}\text { Expressway } \\
\text { Mini Expression }\end{array}$ & $\begin{array}{c}\text { Expressway } \\
\text { Mini Expression }\end{array}$ & $\begin{array}{c}\text { Cell-free } \text { E. coli } \\
\text { Protein } \\
\text { Synthesis }\end{array}$ & $\begin{array}{c}\text { E. coli } \text { S30 } \\
\text { Extract System }\end{array}$ \\
\hline Shaking & $\mathrm{N}$ & $\mathrm{N}$ & $\mathrm{Y}$ & $\mathrm{Y}$ & $\mathrm{Y}$ \\
\hline$[$ Extract] $(\% \mathrm{v} / \mathrm{v})$ & - & 27 & 40 & 24 & 30 \\
\hline $\begin{array}{c}{[\mathrm{pJL} 1-\mathrm{sfGFP}]} \\
(\mathrm{ng} / \mu \mathrm{L})\end{array}$ & 16 & 16 & 10 & 15.6 & 16 \\
\hline $\begin{array}{c}\text { Temperature } \\
(\mathrm{C})\end{array}$ & 29 & 30 & 37 & 37 & 37 \\
\hline
\end{tabular}

* All reactions performed according to the recommended kit instructions

* All reactions performed in final CFPS reaction volumes of $30 \mu \mathrm{L}$

* $16 \mathrm{ng} / \mu \mathrm{L}$ pJL1-sfGFP template was used if no DNA concentration was recommended

$* 37^{\circ} \mathrm{C}$ reaction temperature was used if no specific reaction temperature was recommended

* ' '’ represents unknown values 


\section{Table S7. Additives Cost}

\begin{tabular}{|c|c|c|c|c|}
\hline Additive & Purchased As & $\begin{array}{l}\text { Purchase Cost } \\
\text { (USD)* }\end{array}$ & $\begin{array}{l}\text { Concentration } \\
\text { per } 30 \mu \mathrm{L} \text { rxn }\end{array}$ & $\begin{array}{c}\text { Cost per } 30 \mu \mathrm{L} \text { rxn } \\
(\mathrm{USD})^{*}\end{array}$ \\
\hline $\begin{array}{l}\text { Adenosine } \\
\text { Triphosphate }\end{array}$ & $\begin{array}{c}\text { ThermoFisher NTP } \\
\text { Set } \\
(0.25 \mathrm{~mL} \times 100 \mathrm{mM})\end{array}$ & $\$ 137.00 / 4$ & $1.2 \mathrm{mM}$ & $\$ 0.05$ \\
\hline $\begin{array}{l}\text { Polyethylene Glycol } \\
8000\end{array}$ & $\begin{array}{c}\text { Fisher Bioreagents } \\
\text { Polyethylene Glycol } \\
8000(100 \mathrm{~g})\end{array}$ & $\$ 85.00$ & $0.5 \% \mathrm{w} / \mathrm{v}$ & $\$ 1.28 \times 10^{-4}$ \\
\hline Triton X-100 & $\begin{array}{c}\text { Sigma Triton } \mathrm{X}-100 \\
\text { (1 liter) }\end{array}$ & $\$ 77.40$ & $50 \mathrm{CMC}$ & $\$ 1.56 \times 10^{-5}$ \\
\hline Trehalose & $\begin{array}{l}\text { Acros organics D- } \\
\text { trehalose dihydrate } \\
(100 \mathrm{~g})\end{array}$ & $\$ 217.45$ & $20 \mathrm{mM}$ & $\$ 4.47 \times 10^{-4}$ \\
\hline Betaine & $\begin{array}{c}\text { Thermoscientific } \\
\text { Betaine } \\
(10 \mathrm{~mL} \text { x } 5 \mathrm{M})\end{array}$ & $\$ 73.70$ & $330 \mathrm{mM}$ & $\$ 2.21 \times 10^{-4}$ \\
\hline
\end{tabular}

* Tax not included

* All listed concentrations are representative of the supplemented concentration, not necessarily the total reaction concentration 
Note S1. All raw data (pre-Grubbs' test) can be found in the supporting file named "PreGrubbs' Raw Data.xlsx". 